\title{
Reubicación del parque de transformadores de los sistemas de distribución de Bogotá D.C. mediante algoritmos genéticos
}

\author{
Relocation of electric transformers lot in Bogotá \\ distribution systems using genetic algorithms
Johnn Alejandro Quintero Salazar ${ }^{1} \quad$ Alexander Molina Cabrera ${ }^{2}$
Edwin Andrés Quintero Salazar ${ }^{2}$ \\ Recibido 30 de septiembre de 2011, aceptado 16 de marzo de 2012 \\ Received: September 30, $2011 \quad$ Accepted: March 16, 2012
}

\begin{abstract}
RESUMEN
Este documento presenta una metodología basada en algoritmos genéticos que permite adelantar el reordenamiento del parque de transformadores existente en el nivel de tensión I de los sistemas de distribución de Bogotá D.C., Colombia, con el fin de maximizar el reconocimiento de activos que el ente regulador CREG (Comisión Reguladora de Energía y Gas) realiza a los distintos operadores de red, según lo establecido en la resolución 097 de 2008. Para la aplicación del algoritmo se obtuvieron mediciones de potencia activa máxima para cada hora del año 2009 en un conjunto de 94 transformadores de diferentes capacidades, escogidos de forma aleatoria, instalados en el sistema de distribución de CODENSA S.A. ESP, empresa encargada de prestar el servicio de energía eléctrica en la ciudad de Bogotá D.C. Con esta información se construyeron curvas de carga diarias representativas y se elaboró una base de datos que contiene los costos operativos del movimiento de los equipos y de las tarifas, a partir de los cuales fue posible modelar la función objetivo y las restricciones del problema, obteniéndose un elevado número de combinaciones posibles (alrededor de $1 * 10^{134}$ ) debido al gran número de nodos y de transformadores presentes en el sistema de distribución. La búsqueda convencional de una solución en la anterior situación implicaría el empleo de tiempos prohibitivos, por lo cual se implementó un algoritmo genético clásico, obteniéndose de esta manera una solución óptima que ofrece una ganancia económica en el primer año, asociada al incremento en el cargo por uso, de \$253.446.362,47 (COP), ganancias que podrían verse incrementadas considerablemente al ejecutar el algoritmo en parques de transformadores más grandes.
\end{abstract}

Palabras clave: Técnicas metaheurísticas, algoritmo genético, curva de carga, cargabilidad, resolución CREG 097 de 2008, CREG (Comisión Reguladora de Energía y Gas).

\section{ABSTRACT}

This paper presents a methodology based on genetic algorithms that allows the reordering of the existing park transformers voltage level I of the distribution systems of Bogotá city, Colombia, in order to maximize the recognition of assets that the regulator CREG (Comisión Reguladora de Energía y Gas) made to the various network operators, as set out in resolution 097 of 2008. For the application of the algorithm, we obtained maximum active power measurements for each hour of the year 2009 in a number of transformers of different capacities, chosen at random, installed in the distribution system CODENSA SA ESP, the company that provides electric service in the Bogotá city. With this information we built representative daily load curves and developed a database that contains the operating costs of moving equipment and tariffs, from which it was possible to model the objective function and constraints of the

\footnotetext{
1 Gerencia de Distribución. CODENSA S.A. ESP. Carrera 13ª N $^{\circ}$ 93-66. Bogotá D.C., Colombia. E-mail: jquinteros@ codensa.com.co

2 Programa de Ingeniería Eléctrica. Universidad Tecnológica de Pereira. Complejo Educativo La Julita. Pereira, Colombia. E-mail: almo@utp.edu.co; equintero@utp.edu.co
} 
problem, obtaining a high number of possible combinations (about $1 * 10^{134}$ ) due to the large number of nodes and processors present in the distribution system. Conventional search of a solution in the above situation implies the use of prohibitive times. For this reason we implemented a classical genetic algorithm, thus obtaining an optimal solution that offers a financial gain in the first year, associated with the increase in the usage charge, of \$253.446.362,47 (COP), profits that could be increased considerably when running the algorithm on larger transformers parks.

Keywords: Metaheuristic techniques, genetic algorithms, load curve, chargeability, CREG Resolution 097 of 2008, CREG (Comisión Reguladora de Energía y Gas).

\section{INTRODUCCIÓN}

El sector eléctrico en Colombia está constituido por un conjunto de empresas encargadas de generar, transmitir, comercializar y distribuir la energía eléctrica a los usuarios finales para fines industriales, comerciales o residenciales. A la vez estos usuarios deben pagar por cada $\mathrm{kW} / \mathrm{h}$ consumido de acuerdo a un conjunto de variables establecidas por un ente regulador conocido como CREG (Comisión de Regulación de Energía y Gas) [3].

La etapa de distribución de energía eléctrica está compuesta por los sistemas de transmisión regional (STR) y los sistemas de distribución local (SDL). Los primeros consisten en redes regionales o interregionales de transmisión, conformadas por un conjunto de líneas y subestaciones con sus equipos asociados y que operan a tensiones menores de 220 $\mathrm{kV}$, sin pertenecer a un sistema de distribución local. Entre tanto, los SDL son sistemas de transmisión de energía eléctrica compuestos por redes de distribución municipales o distritales, conformadas por el conjunto de líneas y subestaciones, con sus equipos asociados, que operan a tensiones menores de $220 \mathrm{kV}$, y sin pertenecer a un sistema de transmisión regional, ya que están dedicadas al servicio de un sistema de distribución municipal, distrital o local [4].

La tarifas por concepto de distribución están basadas en el principio de suficiencia financiera definido en los artículos 44 de la ley 143 de 1994 y 87 de la ley 142 de 1994 , los cuales expresan que se debe garantizar a las empresas eficientes la recuperación de sus costos de inversión y sus gastos de administración, operación y mantenimiento [5]. Además, el Estado ejerce control sobre los operadores de red con el fin de que la ineficiencia sea asumida por las empresas de distribución y no por los usuarios. Para efectuar tal control, fue creada la CREG (Comisión de Regulación de Energía y Gas), la cual es una comisión técnica adscrita al Ministerio de Minas y Energía, encargado de establecer los precios del $\mathrm{kW} / \mathrm{h}$, asegurando la viabilidad financiera del negocio de la distribución, reconociendo las diferencias zonales que hacen incurrir en diferentes costos a las empresas que realizan el suministro (dispersión geográfica de la carga suministrada, distribución mediante cables aislados en zonas urbanas en lugar de conductores aéreos en zonas rurales, impacto del clima o de los accidentes geográficos del terreno, etc.) [6]. Además de las anteriores tareas descritas, la CREG determina la remuneración asociada a los costos eficientes de distribuir la energía eléctrica con unos requisitos de calidad del servicio y de pérdidas técnicas establecidos [7].

El 26 de septiembre de 2008 fue expedida la resolución 097 [2], en la cual se actualizan los criterios para el cobro del kW/h en los diferentes niveles de tensión, añadiendo un nuevo capítulo con el nombre de "Criterio de eficiencia para transformadores del nivel de tensión I', con el cual se restringió la retribución de transformadores pertenecientes a este nivel de tensión a la cargabilidad que posean. Así, si el factor de carga es de $40 \%$ o superior, la retribución se hace totalmente. $\mathrm{Si}$, por el contrario, el factor de carga está por debajo del $40 \%$ entonces el reconocimiento de este activo se haría con respecto a la capacidad nominal de la unidad constructiva que instalada en la misma carga presentara un factor superior al $40 \%$ [2].

Actualmente, según estudios realizados en diferentes sistemas de distribución de Colombia, el conjunto de transformadores pertenecientes al nivel de tensión I presenta un alto grado de sobredimensionamiento [8] debido principalmente a dos factores. El primero consiste en que su dimensionamiento se basa en 
la norma NTC 2050 [9], la cual es una traducción directa de las normas estadounidenses, sin tener en cuenta que el consumo de los usuarios en los dos países puede ser diferente [4]. El segundo factor consiste en la reglamentación vigente hasta el año 2008, según la cual el reconocimiento de activos a las empresas de distribución no dependía de su eficiencia (resolución 082 de 2002) [6].

Según lo planteado en párrafos anteriores, una situación problemática actual consiste en que las empresas de distribución no cuentan con una metodología eficiente que les permita una gestión adecuada del parque de transformadores instalados en sus sistemas de distribución pertenecientes al nivel de tensión I [10], simplemente porque las técnicas convencionales no ofrecen resultados confiables dado el elevado número de combinaciones posibles (cerca de $1 * 10^{134}$ ). Así pues, en el presente documento se plantea una metodología basada en un algoritmo genético que posibilita la obtención de una solución óptima al problema de la reubicación de los transformadores de distribución, la cual les permitirá a los operadores de red un mayor reconocimiento de activos por parte de la CREG, incrementando de esta manera la eficiencia del sistema eléctrico colombiano.

\section{INCREMENTO EN FACTURACIÓN POR CONCEPTO DE DISTRIBUCIÓN (X1)}

Los sistemas de transmisión regional y/o distribución local se clasifican por niveles en función de la tensión nominal de operación según se observa en la Figura 1.

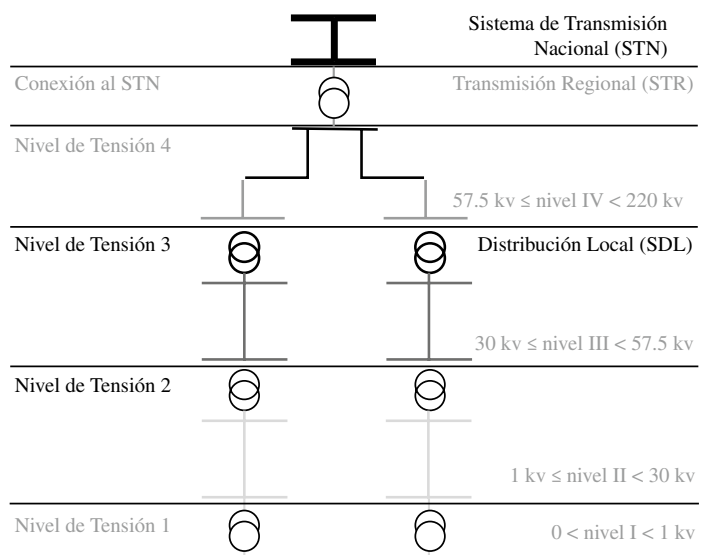

Figura 1. Niveles de tensión del sistema de distribución colombiano.
El Estado colombiano a través de la ley 142 de 1994 [2] estableció los criterios para la determinación de tarifas, considerando criterios de eficiencia económica, neutralidad, solidaridad, redistribución, suficiencia financiera, simplicidad y transparencia, garantizando la recuperación de los costos y gastos de operación, incluyendo expansión, reposición y mantenimiento, permitiendo así remunerar el patrimonio de los accionistas.

El procedimiento para establecer el cargo por concepto de distribución $(D)$ se encuentra relacionado en la ecuación (1). Este es diferente para cada nivel de tensión.

$$
D t_{j, 1, m}=\frac{C D_{4, R, m}}{1-P R_{1, j}}+\frac{C D_{j, 2, m}}{1-P R_{(1,2) j}}+C D I_{j, 1, m}+C D M_{j, 1, m}+\Delta D t_{j, n, m}
$$

Donde $D t_{j, 1, m}$ es el cargo por uso del nivel de tensión I $(\$ / k W h)$, del $O R(j)^{3}$ para el mes $m . C D_{4, R, m}$ y $C D_{j, 2, m}$ son los cargos máximos para el nivel de tensión IV $(\$ / k W h)$ del $S T R^{\circledR}$ y nivel de tensión II respectivamente, correspondientes al mes $m$, del $O R(j)$ establecidos en el capítulo III de la resolución 097 de 2008 [1]. $C D I_{j, 1, m}$ es el cargo máximo del nivel de tensión I, por concepto de inversión del $O R(j)$ en el mes $m . C D M_{j, 1, m}$ es el cargo máximo del nivel de tensión I, por concepto de administración operación y mantenimiento (AOM), para redes del nivel de tensión I del $O R(j)$ en el mes $m . P R_{l, j}$ es el factor para referir las medidas de energía del nivel de tensión I al STN en el sistema del $O R(j)$, según lo definido en el capítulo XII de la misma resolución [2]. $P R_{(1,2), j}$ es el factor para referir las medidas de energía del nivel de tensión I al nivel de tensión II del $O R(j)$, según lo definido en el capítulo mencionado anteriormente. $D t j, n, m$ es el incentivo por variación trimestral de la calidad para el $O R(j)$ durante el mes $m$, aplicable al cargo por uso del nivel de tensión $n$, en $\$ / k W h$, de acuerdo con lo establecido en el capítulo XI de mencionada resolución [2].

Analizando la ecuación (1) se encontró que los únicos términos que variaban al realizar la reubicación del conjunto de transformadores eran $C D I_{j, 1, m}$ y $C D M_{j, 1, m}$, mientras que los demás permanecerían constantes

\footnotetext{
3 En el presente documento, el término $O R(j)$ hace referencia al operador de red, que para el caso de Bogotá D.C. es CODENSA S.A. ESP.
} 
debido a que son independientes del incremento en el reconocimiento de activos en el nivel de tensión I. Así pues, el cargo por uso del nivel de tensión I $\left(D t_{j, 1, m}\right)$ se puede expresar mediante la ecuación (2).

$$
D_{t j, 1, m}=A+C D I_{j, 1, m}+C D M_{j, 1, m}
$$

Con:

$$
A=\frac{C D_{4, R, m}}{1-P R_{1, j}}+\frac{C D_{j, 2, m}}{1-P R_{(1,2) j}}+\Delta D_{t j, n, m}
$$

Donde la constante $A$ agrupa los términos que no dependen del reconocimiento de activos.

En busca de encontrar la utilidad financiera asociada a la ejecución del proyecto, y en vista de que el cargo por uso para el nivel de tensión I se calcula a partir de una muestra representativa de redes y transformadores pertenecientes a este nivel de tensión, se supuso un sistema de distribución ficticio para el cual el conjunto de 94 transformadores de la muestra constituyeran la totalidad de transformadores instalados en el nivel de tensión I. Así, el beneficio obtenido una vez realizada la reubicación de los transformadores será igual a la diferencia entre cargos por uso encontrados para el sistema de distribución ficticio antes y después de ejecutado el proyecto según la ecuación (4).

$$
\Delta D=D t_{1}-D t_{2}
$$

Donde $\Delta D$ es la variación del cargo por uso $(\$ / k W h)$ asociada al reordenamiento de los transformadores, $D t_{l}$ es el cargo por uso del nivel de tensión I $(\$ / k W h)$ encontrado para el sistema de distribución ficticio según la disposición inicial de los transformadores, y $D t_{2}$ es el cargo por uso del nivel de tensión I $(\$ / k W h)$ encontrado para el sistema de distribución ficticio una vez realizada la reubicación.

Reemplazando la ecuación (3) en (4) se construye la ecuación (5).

$$
\Delta D=\left(A+C D I_{2}+C D M_{2}\right)-\left(A+C D I_{1}+C D M_{1}\right)
$$

Con:

$$
\begin{gathered}
\triangle C D I=C D I_{2}-C D I_{1} \\
\triangle C D M=C D M_{2}-C D M_{1}
\end{gathered}
$$

Donde $\triangle C D I$ y $\triangle C D M$ representan la variación en cargo máximo del nivel de tensión I por concepto de inversiones y el cambio en el cargo máximo del nivel de tensión I por concepto de administración, operación y mantenimiento respectivamente. $C D I_{l}$ y $C D I_{2}$ representan los cargos máximos en el nivel de tensión I por concepto de inversiones encontrados antes y después de la reubicación respectivamente. $C D M_{1}$ y $C D M_{2}$ son los cargos máximos del nivel de tensión I por concepto de administración, operación y mantenimiento hallados antes y después de realizada la reubicación de los transformadores.

A partir de las ecuaciones (5), (6) y (7), la variación en el cargo por uso $(\Delta D)$ se reduce a la ecuación (8).

$$
\Delta D=\Delta C D I+\Delta C D M
$$

El cargo por concepto de inversión $(C D I)$ para el nivel de tensión I es calculado a partir de una muestra representativa de redes y transformadores pertenecientes a este nivel de tensión, mediante la clasificación de las redes según el sitio y tipo de instalación del transformador (aéreo, subterráneo, urbano, rural), para luego realizar una tipificación de las redes de baja tensión con base en la longitud y demanda de energía. Este factor es calculado de acuerdo a las ecuación (9) establecida en la resolución 097 de 2008 [2].

$$
C D I_{j, 1}=\left(C D I R_{j, 1} * 0,9\right)+\left(\frac{C A I_{j, 1}}{V_{j, 1}+P N T_{j, n r}} * 0,1\right)-\left(\frac{O I_{j}}{V_{j, 1}+P N T_{j, n r}}\right)
$$

Donde $C D I_{j, 1}$ es el cargo máximo por concepto de inversiones para el nivel de tensión I, para el $O R(j)$. $C D I R_{j, 1}$ es el costo unitario de referencia a partir del cual se calcula el costo para remunerar la inversión de los activos del nivel de tensión I, aprobado por la $C R E G$ para el $O R(j)$. Este valor se define como un número fijo en la resolución particular de cada $O R(j) . C A I_{j, 1}$ es el costo anual de los activos de uso en el nivel de tensión I, para el $O R(j)$, definido en el capítulo II de la resolución 097 de 2008 [1]. $O I_{j}$ son otros ingresos por la explotación de los activos remunerados mediante cargos por uso en actividades distintas a la de distribución de energía eléctrica. Este valor corresponde al $33 \%$ del valor de los ingresos por este concepto durante el año que finaliza en la fecha de corte. $V_{j, 1}$ son las ventas anuales de energía 
en el nivel de tensión I, efectuadas por todos los comercializadores de energía en el sistema del $O R(j)$. Finalmente, $P N T_{j, n r}$ son las pérdidas no técnicas no reconocidas al $O R(j)$, en $\mathrm{kW} / \mathrm{h}$-año, determinadas de acuerdo con el capítulo XII de la resolución 097 de 2008 [2].

Analizando la ecuación (9) se encontró que al incrementar las cargabilidades de los transformadores pertenecientes al nivel de tensión I el único término que varía es el costo anual de los activos de uso en el nivel de tensión I (CAI), mientras que los demás términos permanecen constantes ya que dependen de otros factores diferentes al reconocimiento de activos, reduciendo el cargo por concepto de inversión $\left(C D I_{j, 1}\right)$ a la ecuación (10).

$$
C D I_{j, 1}=B+\left(\frac{C A I_{j, 1}}{V_{j, 1}+P N T_{j, n r}} * 0,1\right)
$$

Con:

$$
B=\left(C D I R_{j, 1} * 0,9\right)-\left(\frac{O I}{V_{j, 1}+P N T_{j, n r}}\right)
$$

Donde la constante $B$ agrupa los términos independientes del reconocimiento de activos.

Luego, reemplazando la ecuación (10) en (6), se obtiene que la variación en el cargo por concepto de inversiones para el nivel de tensión I $(\triangle C D I)$, queda expresada mediante la ecuación (12).

$$
\Delta C D I=0,1 *\left(\frac{C A I_{1}-C A I_{2}}{V_{J, 1}+P N T_{j, n r}}\right)
$$

El costo anual de los activos de uso en el nivel de tensión I (CAI) se calcula según la resolución 097 de 2008 [2] mediante la ecuación (13).

$C A I_{j, 1}=N T_{j} *\left[\sum_{h=1}^{H}\left(\operatorname{Inv}_{H R j, h} * W_{j, h} * \frac{r}{1-(1+r)^{-v i}}+D\right]\right.$ $D=\sum_{h=1}^{H} \operatorname{In} v_{H T j, h} * W_{j, h} * \frac{r}{\left(1-(1+r)^{-v i}\right.}$

Donde $N T_{j}$ es el número de transformadores de distribución de nivel de tensión I, para el $O R(j)$, sin incluir los transformadores exclusivos de alumbrado público. Inv $v_{H R j, h}$ es la inversión media de redes por circuito estimada en el nivel de tensión I, para el estrato $h$, de la muestra del $O R(j) . \operatorname{In} v_{H T j, h}$ es la inversión media de transformadores por circuito estimada en el nivel de tensión I, para el estrato $h$, de la muestra del $O R(j) . W_{j, h}$ es la ponderación del estrato $h$ de la muestra del $O R(j)$. $r$ es la tasa de retorno reconocida para la metodología de precio máximo, y $V_{i}$ es la vida útil en años, reconocida para los activos $i$ de nivel de tensión I.

En vista de que el reconocimiento de activos por concepto de redes permanecerá constante una vez realizada la reubicación de transformadores, la ecuación (13) se reduce a la ecuación (14).

$$
\begin{gathered}
C A I_{J, 1}=N T_{j} *\left[C+\sum_{h=1}^{H}\left(\operatorname{Inv}_{H T j, h} * W_{j, h} * \frac{r}{1-(1+r)^{-v i}}\right]\right. \\
C=\sum_{h=1}^{H}\left(\operatorname{Inv}_{H R j, h} * W_{j, h} * \frac{r}{1-(1+r)^{-v_{i}}}\right.
\end{gathered}
$$

Donde la constante $C$ agrupa el reconocimiento de activos en lo que a redes se refiere.

Posteriormente, reemplazando la ecuación (14) en (6), la variación en el cargo por concepto de inversiones para el nivel de tensión I $(\triangle C D I)$ queda expresada mediante la ecuación (16).

$$
\begin{aligned}
& \Delta C D I=0,1 * \\
& \left(\frac{\left(N T_{J} * \sum_{h=1}^{H}\left(I n v_{H T_{1}} * W_{j, h} * \frac{r}{1-(1+r)^{-v_{i}}}\right)\right)-F}{V_{j, 1}+P N T_{j, n r}}\right) \\
& F=\left(N T_{j} * \sum_{h=1}^{H}\left(I n v_{H T_{2}} * W_{j, h} * \frac{r}{1-(1+r)^{-v_{i}}}\right)\right.
\end{aligned}
$$

El factor de ponderación del estrato $h$, de la muestra del $O R(j)$, se obtiene mediante la ecuación 4.35 definida en el capítulo $\mathrm{V}$ de la resolución 097 de 2008 [2].

$$
W_{j, h}=\frac{N_{j, h}}{N T_{j}}
$$


El término Vi (vida útil) se toma de la Tabla 1, tomada de la resolución 097 de 2008 [2].

Tabla 1. Vida útil reconocida. Resolución 097 de 2008.

\begin{tabular}{|l|c|}
\hline \multicolumn{1}{|c|}{ Activos } & Años \\
\hline Redes & 30 \\
\hline Transformadores & 20 \\
\hline
\end{tabular}

La tasa de retorno reconocida para la metodología de precio máximo $(r)$ toma un valor de $9 \%$ [1].

La inversión media de transformadores por circuito $\left(I n v_{H T j, h}\right)$ se determina mediante la ecuación (18) descrita en el capítulo $V$ de [2].

$$
\operatorname{In} v_{H T j}, h=\frac{1}{N M_{j, h}} * \sum_{h=1}^{N M_{j, h}} I n v_{C_{j, h, k}}
$$

Donde $I n v_{C j, h, k}$ es la inversión estimada en el transformador del circuito $k$ del estrato $h$, de la muestra del $O R(j) . N M_{j, h}$ es el número de transformadores de distribución de nivel de tensión I, de la Muestra del $O R(j)$, del estrato $h$.

Para obtener la inversión estimada en el transformador del circuito $k$ del estrato $h\left(I n v_{C j, h, k}\right)$ se utiliza el inventario de los circuitos de la muestra reportados por el $O R(j)$ a la CREG en respuesta a la circular CREG 013 de 2007 [7], y los costos reconocidos en el numeral 5.3 de [1] (ver Tabla 2), aplicando los criterios definidos en el capítulo XIV de [1] donde se especifica que el valor a reconocer depende de la cargabilidad. Así, si esta se encuentra por encima del $40 \%$, su reconocimiento se hace totalmente, mientras que si se ubica por debajo el reconocimiento se hace con respecto al transformador que instalado en dicha carga presente una cargabilidad igual o inmediatamente superior al $40 \%$.

Si Fct $t_{i} \geq 0,4$ entonces CapT $_{i}=\operatorname{CapR}_{i}$

Si Fct $t_{i}<0,4$ entonces CapT $T_{i}=\operatorname{Com}_{i}\left\{\frac{F c t_{i}}{0,4}\right\}$

Con:

$$
\operatorname{CapA}_{i}=\operatorname{CapR}_{i} *\left(\frac{F c t_{i}}{0,4}\right)
$$

Donde $F_{c t i}$ es la cargabilidad estimada del transformador $i, \operatorname{Cap}_{i}$ es la capacidad del transformador $i(k V A)$ a reconocer, $\operatorname{CapR}_{i}$ es la capacidad del transformador $i(k V A)$ reportada por el $O R(j), C a p A_{i}$ es la capacidad de un transformador $(k V A)$ cargado al $40 \%$ considerando la demanda de energía anual del transformador $i, \operatorname{Com}_{i}\left(\operatorname{CapA}_{i}\right)$ es la capacidad del transformador monofásico o trifásico $(k V A)$ inmediatamente superior al valor de $\operatorname{Cap} A_{i}$ según la Tabla 2 publicada en la resolución 097 de 2008 [2].

Una vez encontrada la variación en el cargo por uso asociada a la reubicación de transformadores $(\Delta D)$, el beneficio obtenido asociado a la ejecución del proyecto estará dado por el incremento de la tarifa, multiplicado por la energía consumida durante un año en el conjunto de transformadores [11], según la ecuación (20).

$$
X_{1}=\left(\Delta D * T E_{\text {ere }} * N_{\text {años }}\right)
$$

con:

$$
T E_{\text {ere }}=\sum_{i=1}^{94} T E_{\text {ere }_{i}}
$$

donde $(\Delta D)$ es la variación en el cargo por uso del nivel de tensión I para el conjunto de transformadores, $T E_{\text {ere }_{i}}$ es la energía anual demandada en el conjunto de cargas, $N_{a n ̃ o s}$ es el tiempo en años en que se evalúa

\begin{tabular}{|c|c|c|c|}
\hline $\mathbf{N}^{\circ}$ Fases & 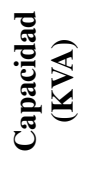 & $\stackrel{\varrho}{\equiv}$ & $\stackrel{\hat{\theta}}{\stackrel{\theta}{0}}$ \\
\hline Trifásico & 15 & Aéreo & 2.606 .440 \\
\hline Trifásico & 20 & Aéreo & 3.110 .233 \\
\hline Trifásico & 30 & Aéreo & 3.718 .805 \\
\hline Trifásico & 45 & Aéreo & 4.619 .170 \\
\hline Trifásico & 50 & Aéreo & 4.920 .960 \\
\hline Trifásico & 75 & Aéreo & 5.429 .900 \\
\hline Trifásico & 113 & Aéreo & 6.695 .315 \\
\hline Trifásico & 150 & Aéreo & 10.956 .730 \\
\hline
\end{tabular}
el proyecto, y $E_{\text {ere }_{i}}$ es la energía anual demandada en la carga $i$.

Tabla 2. Precios reconocimiento de transformadores.

Fuente: CREG 097 de 2008 (\$COP). 
Las anteriores definiciones fueron implementadas en el entorno Matlabß. La variación del cargo por uso $(\Delta D)$ es calculada para un conjunto de combinaciones posibles, según la búsqueda ejecutada por el algoritmo genético implementado, descrito posteriormente en este documento.

\section{Inversión (X2)}

La inversión en el proyecto está dada por la suma de los costos asociados al retiro e instalación de algunos transformadores del conjunto, los cuales dependen de la capacidad de los mismos (ver Tabla 3). Este gasto se encontró a partir de la comparación entre las capacidades de los transformadores instalados en las cargas antes y después del reordenamiento, contenidas éstas en los vectores $J_{o}$ y $J_{f}$ respectivamente. Así, si la capacidad no coincide en la misma posición de los dos vectores, se suma el retiro del transformador existente en el vector $J_{o}$ y la instalación de la capacidad que aparece en la misma posición del vector $J_{f}$. Entre tanto, si la capacidad coincide, entonces no se suma ningún valor, tal y como se expone en la ecuación (22).

$$
\begin{aligned}
& X_{3}=\sum_{k=1}^{94} R_{i_{0}}+I_{i_{f}} \\
& \forall i \in J_{0}(i, 1) \neq J_{f}(i, 1)
\end{aligned}
$$

Tabla 3. Precios de retiro e instalación de transformadores trifásicos 2009.

\begin{tabular}{|c|c|c|}
\hline $\begin{array}{c}\text { Capacidad } \\
\text { (KVA) }\end{array}$ & \$ Retiro & \$ Instalación \\
\hline 15 & 129.682 & 129.682 \\
\hline 30 & 129.682 & 129.682 \\
\hline 45 & 129.682 & 129.682 \\
\hline 75 & 129.682 & 129.682 \\
\hline 113 & 129.682 & 129.682 \\
\hline 150 & 258.959 & 258.959 \\
\hline 225 & 258.959 & 258.959 \\
\hline 300 & 258.959 & 258.959 \\
\hline
\end{tabular}

Fuente: CODENSA (\$COP).

Donde $R_{i o}$ es el costo en pesos del retiro del transformador instalado inicialmente en la carga $i, I_{i f}$ es el costo en pesos de la instalación del transformador en la carga $i$ una vez realizada la reubicación, $J_{0}$ es el vector que contiene las capacidades de los transformadores instalados en las cargas inicialmente, y $J_{f}$ es el vector que contiene las capacidades de los transformadores instalados en las cargas una vez realizado el reordenamiento de los mismos. $\mathrm{El}$ anterior procedimiento fue implementado en entorno Matlab®.

\section{MODELO MATEMÁTICO}

Según lo expuesto, el problema consiste en reordenar el conjunto de transformadores, optimizando una función de costos según la ecuación (23) [12], donde las ganancias corresponden al incremento en la facturación por concepto de distribución $\left(X_{I}\right)$ y los egresos serán iguales a la inversión que se deba hacer para realizar la instalación o retiro de algunos transformadores $\left(X_{2}\right)$.

$$
\text { Función Objetivo }=X_{1}-X_{2}
$$

Las restricciones del problema son de dos tipos. $\mathrm{La}$ primera de ellas corresponde al número máximo de transformadores de cada capacidad, el cual debe corresponder con el estado inicial. La segunda se refiere a la cargabilidad de los mismos, ya que si bien es cierto que se quiere incrementar sus cargabilidades, estas no deben sobrepasar el $90 \%$ con el fin de que no se afecte su vida útil de 20 años según lo estipulado en las normas NTC 818 y 819 [5].

\section{Restricción de cargabilidad Fci $\leq 90 \%$}

Donde $F c i$ es la cargabilidad del transformador $i$. Por otra parte, la restricción existente sobre número de transformadores de cada capacidad viene dada por:

$$
\left(N^{\circ} T \operatorname{Car} R\right)_{\mathrm{o}}=\left(N^{\circ} T \operatorname{Car} R\right)_{f}
$$

Donde $\left(N^{\circ} T \text { CarR }\right)_{0}$ es el número de transformadores de capacidad $R$ en el conjunto inicial, y $\left(N^{\circ} T C a r R\right)_{f}$ es el número de transformadores de capacidad $R$ en el conjunto una vez realizado el reordenamiento.

\section{ALGORITMO GENÉTICO}

Debido a la gran cantidad de combinaciones posibles a la hora de realizar el reordenamiento del conjunto de transformadores, se hizo necesario el uso de técnicas heurísticas [13], las cuales sacrifican la garantía de encontrar el resultado óptimo a cambio de obtener una buena solución en un tiempo razonable [14]. 
En la Figura 2 es posible apreciar el diagrama de flujo del algoritmo genético.

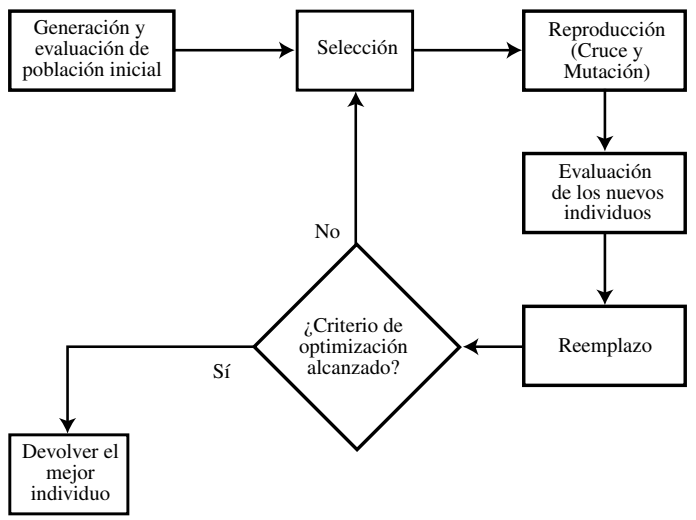

Figura 2. Diagrama de flujo del algoritmo genético.

\section{Creación de población inicial}

La implementación de este algoritmo está basada en numeración binaria. Por este motivo se hizo necesario establecer inicialmente una codificación de los transformadores según su capacidad, tal como se muestra en la Tabla 4. De esta manera el transformador instalado en la carga $i$ se representa por 3 bits que varían dependiendo su capacidad. El individuo que representa la disposición de todos los transformadores sobre las 94 cargas está compuesto entonces por 282 bits.

Tabla 4. Codificación de transformadores según capacidad.

\begin{tabular}{|c|c|c|}
\hline $\begin{array}{c}\text { Capacidad } \\
\text { (KVA) }\end{array}$ & Código & $\begin{array}{c}\mathbf{N}^{\circ} \\
\text { Transformadores }\end{array}$ \\
\hline 75 & 000 & 13 \\
\hline 113 & 001 & 17 \\
\hline 150 & 010 & 26 \\
\hline 225 & 011 & 14 \\
\hline 300 & 100 & 9 \\
\hline 400 & 101 & 4 \\
\hline 500 & 110 & 11 \\
\hline
\end{tabular}

La creación de la población inicial se realizó generando un número establecido de individuos de manera aleatoria, cada uno de los cuales es representado por un número binario de 282 bits.

Una vez generada la población inicial se verificó que cada individuo cumpliera con las restricciones de cargabilidad y número máximo de transformadores de cada capacidad. En caso de que no lo hiciera, se realizó un ajuste en el mismo hasta que cumpliera con las restricciones según el diagrama de flujo mostrado en la Figura 3 [15]. Una vez todos los individuos cumplen con las restricciones propuestas en el modelo matemático, se procede con la creación de la población inicial [16].

\section{Selección de individuos mejor adaptados}

La selección busca que los individuos que tengan mejor adaptación (función de adaptación) puedan transmitir su información con mayores probabilidades que los individuos menos adaptados. El valor de la función o valor de adaptación se relaciona íntimamente con la función objetivo y representa la manera como los individuos afrontan la presión del medio ambiente [17].

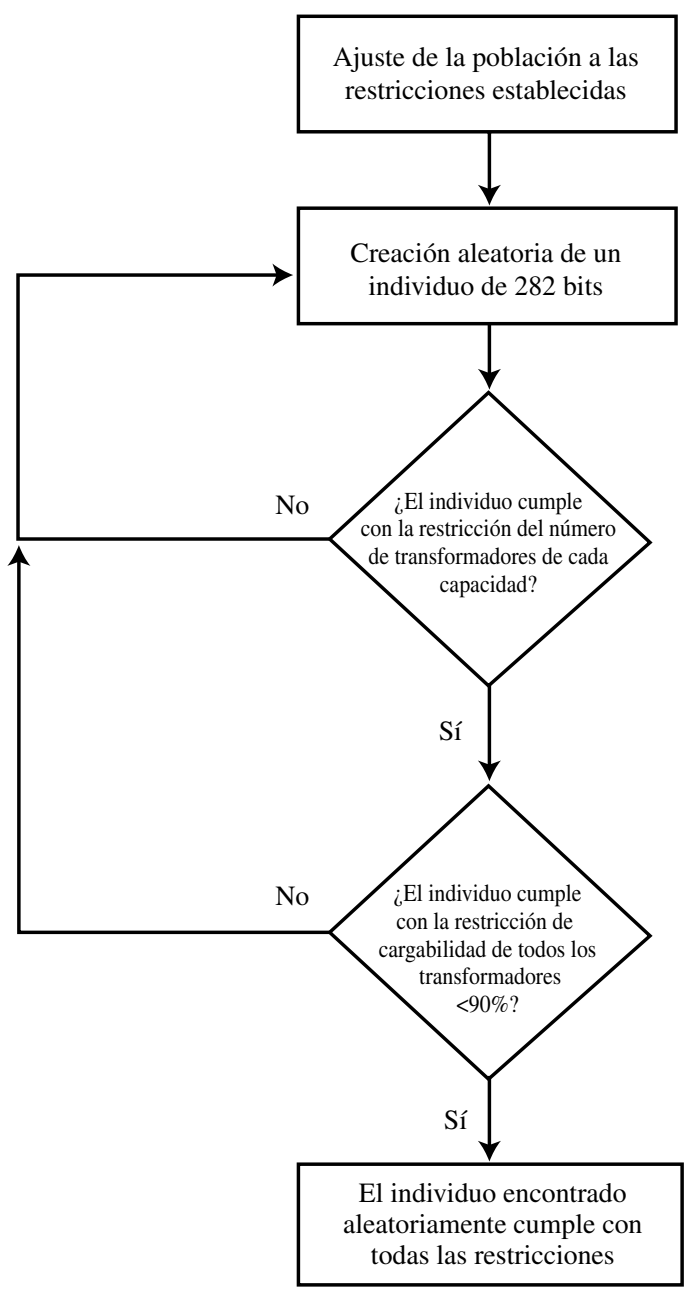

Figura 3. Diagrama de flujo de la función de ajuste de la población de transformadores. 
El proceso de selección está compuesto por dos fases. La primera de ellas busca determinar el valor de adaptación o valor Fitness, mientras que la segunda determina el número de descendientes a los que cada individuo tiene derecho [18].

\section{- Función de adaptación:}

El cálculo del valor de adaptación de cada individuo se realiza mediante las ecuaciones (24) y (25).

$$
\begin{gathered}
Z_{l i}=\frac{F_{l i}}{\sum_{i=1}^{N^{\circ} I n d} F_{l i}} \\
F_{l i}=\frac{f_{i}-L}{U-L}
\end{gathered}
$$

Donde $f i$ es el individuo $i$ evaluado en la función objetivo, $L$ es $90 \%$ del menor valor de función objetivo de la población, y $U$ es el mayor valor de la función objetivo de la población.

\section{- Cálculo de número de descendientes:}

En este caso se usó el método de la ruleta adaptado, en el cual los valores de $Z_{l i}$ representan un porcentaje de la rueda correspondiente a un individuo.

Este método consiste en armar una ruleta imaginaria con valor normalizado y a continuación obtener un número aleatorio entre 0 y 1 , de forma que este sea el que indique el individuo favorecido. Se lanzan tantos números aleatorios como individuos tenga la población. Así, un individuo tendrá una o varias opciones de recombinarse con otro individuo [19].

\section{Recombinación}

La recombinación se realizó en dos etapas. La primera de ellas consistió en establecer de manera aleatoria las parejas a recombinar. La segunda etapa determina el punto de cruce o la porción de material que se va a intercambiar y si finalmente los individuos se reproducen o no [20].

\section{Selección de las parejas a recombinar}

Se generó un número aleatorio entre uno y el número máximo de individuos seleccionados en la etapa anterior. Luego se generó otro número aleatorio con el fin de seleccionar la pareja. Se repitió este proceso hasta agotar el número de individuos y cumplir con el número de descendientes de cada uno establecido anteriormente [21].

\section{Selección del punto de cruce}

Antes de seleccionar el punto de cruce es necesario considerar la tasa de recombinación y decidir si tal pareja se recombina o si se deja intacta para que pase a la siguiente generación. Para esto se lanzó un aleatorio entre 0 y 1 . Si tal número aleatorio era menor a la tasa de recombinación, la pareja se recombinaba, de lo contrario se dejaba intacta [22].

Para seleccionar el punto de cruce es necesario tener en cuenta el número de elementos que componen la información genética de cada individuo (para este caso 282 bits). Ahora, para establecer el punto donde se da el intercambio de información, se lanzó un aleatorio entre 1 y 281 .

\section{Mutación}

La mutación es el proceso de cambio de valores en los alelos, de forma que se introduce nueva información a la población, es decir, nuevas características. De esta forma se incluye mayor posibilidad de encontrar la función óptima [23].

Las posiciones a mutar se establecen de acuerdo a la tasa de mutación, y son seleccionadas aleatoriamente de la matriz de población cambiando el valor del gen escogido de 0 a 1 o viceversa, según sea el caso.

\section{Ajuste y evaluación de los nuevos individuos}

Una vez creada la nueva población se verificó que cada individuo cumpliera con las restricciones de cargabilidad y número máximo de transformadores por capacidad. En caso de que no lo hiciera se realizó un ajuste en el mismo hasta que cumpliera con las restricciones. Luego que el conjunto de individuos cumplió con las restricciones establecidas en el modelo matemático, éstos fueron evaluados en la función objetivo, almacenándose el mejor individuo y continuando a la siguiente iteración.

\section{Criterio de parada}

El criterio de parada seleccionado fue el número de iteraciones. Este fue modificado en repetidas ocasiones junto con las tasas de recombinación y mutación en miras de comparar resultados y obtener la mejor respuesta.

La implementación del algoritmo genético en su conjunto fue realizada en el entorno de simulación integrado Matlab®. 


\section{RESULTADOS}

La matriz de información original tiene un tamaño de 826.440 filas y 11 columnas. En esta matriz se incluyen los consumos de potencia activa horaria para un conjunto de 94 transformadores trifásicos de distintas capacidades, tal como se muestra en la Figura 4, durante todo el año 2009.

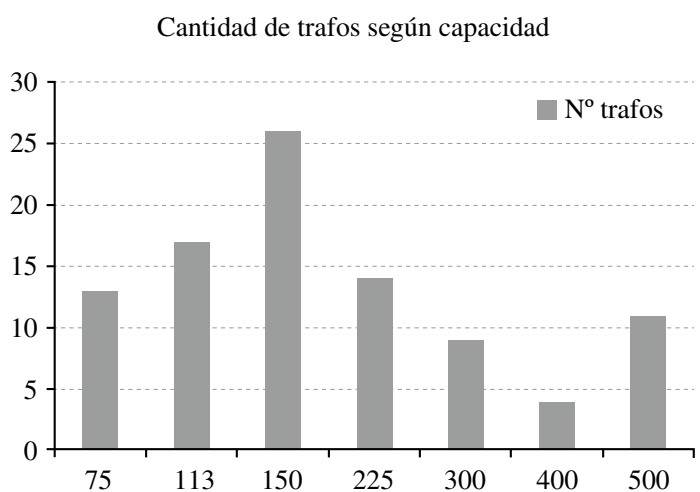

Figura 4. Cantidad de transformadores involucrados en la investigación.

Una vez construida la base de datos con curvas de carga representativas, se encontró que en general, para el conjunto de transformadores, los tres tipos de curvas presentan un comportamiento diferente (ver Figura 5).

Analizando la Figura 5 se aprecia que la curva de carga del tipo de día uno, correspondiente a los días laborales, presenta la cargabilidad más alta, además está incluye dos picos, el primero de ellos entre las 5 y 7 de la mañana y el segundo entre las 6 y 8 de la noche.

Las curvas de carga representan la vida cotidiana de los usuarios y dependen del entorno sociológico del lugar donde estos habitan. Así, estas curvas pueden diferir entre las diferentes ciudades del país.

La energía anual demandada en las cargas, las capacidades de los transformadores instalados inicialmente y las cargabilidades del conjunto de transformadores, fueron calculadas a partir de las curvas de carga encontradas y la ecuación (26), respectivamente.

$$
\left.F_{c}=\frac{E_{\text {ere anual }}}{S_{\text {nom }} * 8760}\right) * 100
$$

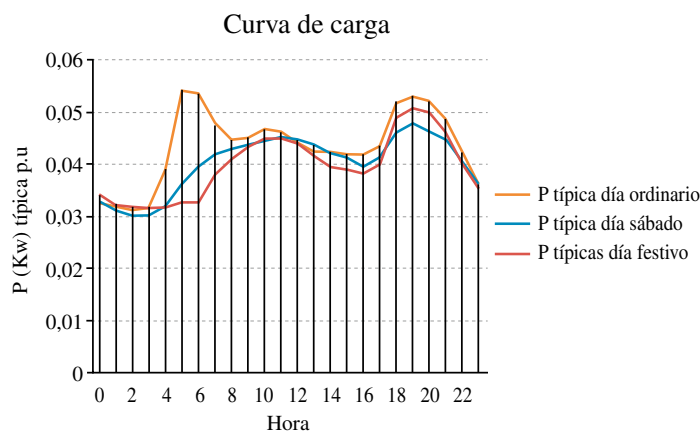

Figura 5. Curvas de carga típicas.

En busca de encontrar la ubicación de los transformadores sobre las cargas que presente el mayor reconocimiento de activos con la menor inversión posible, se implementó un algoritmo genético siguiendo el procedimiento descrito anteriormente. Teniendo en cuenta que este tipo de técnicas sacrifican la garantía de encontrar el resultado óptimo a cambio de obtener una buena solución en un tiempo razonable, y que la búsqueda puede mejorar para unos parámetros de entrada específicos que varían dependiendo el tipo de función a optimizar, se ejecutó el algoritmo en repetidas ocasiones para distintos parámetros de entrada y se almacenó la mejor respuesta en cada caso, utilizando para ello un computador portátil marca Dell $^{\circledR}$, serie Inspiron, con procesador AMD Athlon II P360 (2.3 $G H z$ ), memoria $R A M$ de $2 G B$, y disco duro de 320 $G B$. Los tiempos de ejecución y el mejor individuo de cada iteración evaluado en la función objetivo se resumen en la Tabla 5, donde Población es el número de individuos que componen la población del algoritmo, $T$. $R c$ es la tasa de recombinación,

Tabla 5. Iteraciones realizadas.

\begin{tabular}{|c|c|c|c|c|c|}
\hline 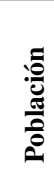 & 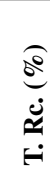 & $\underbrace{\stackrel{\theta}{g}}_{\dot{H}}$ & 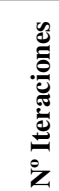 & 产 & 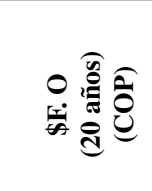 \\
\hline 10 & 70 & 3 & 30 & 01:09:53 & 49.741 .113 \\
\hline 10 & 70 & 15 & 30 & 01:21:20 & 55.968 .429 \\
\hline 10 & 70 & 50 & 30 & 01:18:56 & 43.917 .553 \\
\hline 10 & 10 & 50 & 30 & 01:10:43 & 43.979 .078 \\
\hline 10 & 10 & 15 & 80 & $05: 21: 15$ & 46.680 .204 \\
\hline 10 & 70 & 15 & 80 & 04:32:07 & 46.880 .163 \\
\hline 10 & 70 & 15 & 500 & $23: 50: 53$ & 59.331 .582 \\
\hline
\end{tabular}


T. Mt es la tasa de mutación, y $\$ F . O$ (20 años) son las ganancias asociadas al individuo en 20 años, tiempo elegido para evitar valores negativos.

Después de realizado el conjunto de iteraciones se seleccionó la mejor respuesta obtenida. Esta representa la ubicación de los transformadores sobre las cargas que ofrecen el mayor reconocimiento de activos con la menor inversión, encontrada en un tiempo razonable.

A partir de este individuo seleccionado se calculó nuevamente la cargabilidad de los transformadores con sus nuevas ubicaciones.

Comparando los cuadros de cargabilidad antes (Tabla 6) y después (Tabla 7) de ejecutado el algoritmo, se aprecia que de 52 transformadores que se encontraban con cargabilidad de $40 \%$ o más se pasó a 61. Además se observa que cinco transformadores adicionales se encuentran muy cerca de este umbral, evidenciándose de esta manera que mediante la aplicación del método propuesto se logra incrementar el reconocimiento de activos que la CREG realiza a los operadores de red, en este caso, CODENSA S.A. ESP.

El individuo hallado presenta una variación en el cargo por uso $(\Delta D)$ con respecto a la disposición inicial de:

$$
\Delta \mathrm{D}=0,055636558996729[\$ / \mathrm{kW} / \mathrm{h}](\mathrm{COP})
$$

Esto significa que la tarifa aplicada por la empresa de distribución que opera en la ciudad de Bogotá D.C., una vez aplicada esta metodología al conjunto de 94 transformadores, recaudaría aproximadamente $\$ 0,06$ por cada $k W / h$ consumido por sus suscriptores conectados al nivel de tensión I.

Ahora bien, el número de suscriptores en la ciudad de Bogotá D.C., según lo contenido en el Sistema Único de Información ${ }^{4}$ (SUI), es de 2.148.000. De la misma fuente se obtiene que el consumo promedio de energía eléctrica consumida por usuarios residenciales para este nivel de tensión es de $176,73 \mathrm{~kW} / \mathrm{h}$. A partir de lo anterior, y multiplicando el incremento en la tarifa originado por la aplicación de esta metodología, por el consumo

4 Datos tomados de: www.sui.gov.co. promedio y el número de usuarios, se tiene que las ganancias serían de \$21.120.530,21 (COP) mensuales, lo cual indicaría un ingreso anual de \$253.446.362,47 (COP).

La inversión realizada para realizar la reubicación del conjunto de transformadores $X_{2}$ es:

$$
\mathrm{X}_{2}=\$ 29.789 .608,53(\mathrm{COP})
$$

Tabla 6. Cargabilidad inicial (antes de ejecutado

\begin{tabular}{|c|c|c|c|}
\hline Carga & $\begin{array}{c}\text { Energía Anual } \\
(\mathrm{GW} / \mathrm{h})\end{array}$ & $\begin{array}{c}\text { Cargabilidad } \\
(\%)\end{array}$ & $\begin{array}{c}\text { Capacidad } \\
\text { Trasformador } \\
\text { Instalado (KVA) }\end{array}$ \\
\hline 1 & 2,183 & 49,84 & 500 \\
\hline 2 & 0,338 & 51,54 & 75 \\
\hline 3 & 0,879 & 44,61 & 225 \\
\hline 4 & 0,328 & 49,99 & 75 \\
\hline 5 & 0,267 & 40,64 & 75 \\
\hline 6 & 0,649 & 49,39 & 150 \\
\hline 7 & 1,270 & 128,39 & 113 \\
\hline 8 & 0,158 & 15,96 & 113 \\
\hline 9 & 0,247 & 37,67 & 75 \\
\hline 10 & 0,385 & 29,36 & 150 \\
\hline 11 & 1,723 & 65,57 & 300 \\
\hline 12 & 1,754 & 66,74 & 300 \\
\hline 13 & 1,040 & 79,20 & 150 \\
\hline 14 & 0,629 & 31,93 & 225 \\
\hline 15 & 0,729 & 55,52 & 150 \\
\hline 16 & 0,567 & 57,28 & 113 \\
\hline 17 & 1,423 & 72,22 & 225 \\
\hline 18 & 0,391 & 29,79 & 150 \\
\hline 19 & 0,900 & 68,56 & 150 \\
\hline 20 & 0,294 & 14,93 & 225 \\
\hline 21 & 0,503 & 25,54 & 225 \\
\hline 22 & 0,943 & 71,78 & 150 \\
\hline 23 & 0,483 & 36,75 & 150 \\
\hline 24 & 0,220 & 22,24 & 113 \\
\hline 25 & 0,534 & 12,19 & 500 \\
\hline 26 & 0,766 & 58,32 & 150 \\
\hline 27 & 0,382 & 19,40 & 225 \\
\hline 28 & 0,323 & 32,64 & 113 \\
\hline 29 & 0,513 & 51,82 & 113 \\
\hline 30 & 0,866 & 43,97 & 225 \\
\hline 31 & 0,189 & 19,09 & 113 \\
\hline 32 & 0,924 & 93,42 & 113 \\
\hline 33 & 0,274 & 41,85 & 75 \\
\hline 34 & 0,994 & 75,67 & 150 \\
\hline 35 & 0,616 & 62,26 & 113 \\
\hline 36 & 0,476 & 36,28 & 150 \\
\hline 37 & 0,263 & 40,17 & 75 \\
\hline 38 & 1,451 & 73,63 & 225 \\
\hline
\end{tabular}
el algoritmo genético desarrollado). 


\begin{tabular}{|c|c|c|c|}
\hline Carga & $\begin{array}{c}\text { Energía Anual } \\
\text { (GW/h) }\end{array}$ & $\begin{array}{c}\text { Cargabilidad } \\
(\%)\end{array}$ & $\begin{array}{c}\text { Capacidad } \\
\text { Trasformador } \\
\text { Instalado (KVA) }\end{array}$ \\
\hline 39 & 0,586 & 89,29 & 75 \\
\hline 40 & 0,367 & 37,14 & 113 \\
\hline 41 & 0,299 & 30,23 & 113 \\
\hline 42 & 0,303 & 46,25 & 75 \\
\hline 43 & 0,268 & 20,40 & 150 \\
\hline 44 & 0,858 & 65,32 & 150 \\
\hline 45 & 0,216 & 32,88 & 75 \\
\hline 46 & 0,479 & 48,40 & 113 \\
\hline 47 & 0,436 & 33,24 & 150 \\
\hline 48 & 2,437 & 55,64 & 500 \\
\hline 49 & 0,641 & 48,82 & 150 \\
\hline 50 & 0,566 & 28,73 & 225 \\
\hline 51 & 0,958 & 48,61 & 225 \\
\hline 52 & 0,659 & 50,19 & 150 \\
\hline 53 & 2,204 & 50,32 & 500 \\
\hline 54 & 0,133 & 13,48 & 113 \\
\hline 55 & 1,259 & 35,93 & 400 \\
\hline 56 & 3,689 & 84,22 & 500 \\
\hline 57 & 1,415 & 32,31 & 500 \\
\hline 58 & 1,372 & 52,23 & 300 \\
\hline 59 & 0,678 & 34,40 & 225 \\
\hline 60 & 0,445 & 33,94 & 150 \\
\hline 61 & 0,158 & 15,96 & 113 \\
\hline 62 & 1,727 & 65,73 & 300 \\
\hline 63 & 0,984 & 37,46 & 300 \\
\hline 64 & 2,471 & 70,52 & 400 \\
\hline 65 & 1,745 & 49,82 & 400 \\
\hline 66 & 0,568 & 28,83 & 225 \\
\hline 67 & 0,270 & 41,12 & 75 \\
\hline 68 & 0,520 & 79,20 & 75 \\
\hline 69 & 0,364 & 27,76 & 150 \\
\hline 70 & 2,545 & 58,12 & 500 \\
\hline 71 & 1,309 & 99,66 & 150 \\
\hline 72 & 0,433 & 65,93 & 75 \\
\hline 73 & 2,193 & 83,48 & 300 \\
\hline 74 & 1,290 & 29,46 & 500 \\
\hline 75 & 0,290 & 29,33 & 113 \\
\hline 76 & 1,085 & 82,64 & 150 \\
\hline 77 & 0,192 & 14,67 & 150 \\
\hline 78 & 0,848 & 43,02 & 225 \\
\hline 79 & 0,855 & 24,42 & 400 \\
\hline 80 & 0,262 & 20,00 & 150 \\
\hline 81 & 0,372 & 37,58 & 113 \\
\hline 82 & 1,165 & 88,73 & 150 \\
\hline 83 & 0,539 & 54,49 & 113 \\
\hline 84 & 1,089 & 24,86 & 500 \\
\hline 85 & 0,325 & 24,77 & 150 \\
\hline 86 & 0,423 & 32,20 & 150 \\
\hline 87 & 0,225 & 34,26 & 75 \\
\hline 88 & 0,905 & 45,93 & 225 \\
\hline
\end{tabular}

\begin{tabular}{|c|c|c|c|}
\hline Carga & $\begin{array}{c}\text { Energía Anual } \\
(\mathbf{G W / h})\end{array}$ & $\begin{array}{c}\text { Cargabilidad } \\
(\mathbf{\%})\end{array}$ & $\begin{array}{c}\text { Capacidad } \\
\text { Trasformador } \\
\text { Instalado (KVA) }\end{array}$ \\
\hline 89 & 0,281 & 21,40 & 150 \\
\hline 90 & 1,277 & 48,62 & 300 \\
\hline 91 & 2,217 & 50,63 & 500 \\
\hline 92 & 2,647 & 60,43 & 500 \\
\hline 93 & 0,854 & 32,50 & 300 \\
\hline 94 & 0,481 & 18,30 & 300 \\
\hline
\end{tabular}

Tabla 7. Cargabilidad final encontrada gracias a la implementación del algoritmo genético.

\begin{tabular}{|c|c|c|c|}
\hline Carga & $\begin{array}{c}\text { Energía Anual } \\
\text { (GW/h) }\end{array}$ & $\begin{array}{c}\text { Cargabilidad } \\
(\%)\end{array}$ & $\begin{array}{c}\text { Capacidad } \\
\text { Transformador } \\
\text { Instalado (KVA) }\end{array}$ \\
\hline 1 & 2,183 & 49,84 & 500 \\
\hline 2 & 0,338 & 25,77 & 150 \\
\hline 3 & 0,879 & 66,91 & 150 \\
\hline 4 & 0,328 & 12,49 & 300 \\
\hline 5 & 0,267 & 6,09 & 500 \\
\hline 6 & 0,649 & 32,92 & 225 \\
\hline 7 & 1,270 & 29,01 & 500 \\
\hline 8 & 0,158 & 15,96 & 113 \\
\hline 9 & 0,247 & 37,67 & 75 \\
\hline 10 & 0,385 & 29,36 & 150 \\
\hline 11 & 1,723 & 87,42 & 225 \\
\hline 12 & 1,754 & 66,74 & 300 \\
\hline 13 & 1,040 & 39,60 & 300 \\
\hline 14 & 0,629 & 47,90 & 150 \\
\hline 15 & 0,729 & 73,71 & 113 \\
\hline 16 & 0,567 & 57,28 & 113 \\
\hline 17 & 1,423 & 32,50 & 500 \\
\hline 18 & 0,391 & 59,59 & 75 \\
\hline 19 & 0,900 & 25,71 & 400 \\
\hline 20 & 0,294 & 44,79 & 75 \\
\hline 21 & 0,503 & 50,85 & 113 \\
\hline 22 & 0,943 & 71,78 & 150 \\
\hline 23 & 0,483 & 48,79 & 113 \\
\hline 24 & 0,220 & 16,75 & 150 \\
\hline 25 & 0,534 & 53,95 & 113 \\
\hline 26 & 0,766 & 38,88 & 225 \\
\hline 27 & 0,382 & 8,73 & 500 \\
\hline 28 & 0,323 & 32,64 & 113 \\
\hline 29 & 0,513 & 39,04 & 150 \\
\hline 30 & 0,866 & 87,56 & 113 \\
\hline 31 & 0,189 & 14,38 & 150 \\
\hline 32 & 0,924 & 21,11 & 500 \\
\hline 33 & 0,274 & 20,92 & 150 \\
\hline 34 & 0,994 & 75,67 & 150 \\
\hline 35 & 0,616 & 46,90 & 150 \\
\hline 36 & 0,476 & 72,57 & 75 \\
\hline 37 & 0,263 & 10,04 & 300 \\
\hline 38 & 1,451 & 33,13 & 500 \\
\hline
\end{tabular}




\begin{tabular}{|c|c|c|c|c|c|c|c|}
\hline Carga & $\begin{array}{c}\text { Energía Anual } \\
(\mathrm{GW} / \mathrm{h})\end{array}$ & $\begin{array}{c}\text { Cargabilidad } \\
(\%)\end{array}$ & $\begin{array}{c}\text { Capacidad } \\
\text { Transformador } \\
\text { Instalado (KVA) }\end{array}$ & Carga & $\begin{array}{c}\text { Energía Anual } \\
(\mathrm{GW} / \mathrm{h})\end{array}$ & $\begin{array}{c}\text { Cargabilidad } \\
(\%)\end{array}$ & $\begin{array}{c}\text { Capacidad } \\
\text { Transformador } \\
\text { Instalado (KVA) }\end{array}$ \\
\hline 39 & 0,586 & 44,64 & 150 & 88 & 0,905 & 68,89 & 150 \\
\hline 40 & 0,367 & 55,96 & 75 & 89 & 0,281 & 28,41 & 113 \\
\hline 41 & 0,299 & 15,18 & 225 & 90 & 1,277 & 64,83 & 225 \\
\hline 42 & 0,303 & 15,41 & 225 & 91 & 2,217 & 84,38 & 300 \\
\hline 43 & 0,268 & 40,81 & 75 & 92 & 2,647 & 60,43 & 500 \\
\hline 44 & 0,858 & 43,55 & 225 & 93 & 0,854 & 65,00 & 150 \\
\hline 45 & 0,216 & 6,16 & 400 & 94 & 0,481 & 36,61 & 150 \\
\hline 46 & 0,479 & 48,40 & 113 & & & & \\
\hline
\end{tabular}

\section{CONCLUSIONES}

El comportamiento de la demanda horaria durante un año en el nivel de tensión I presenta una distribución aproximadamente normal dentro de las categorías días laborales, sábados y festivos. Esto permite representar el consumo hora-hora durante el año mediante promedios simples a través de los cuales fue posible construir las curvas correspondientes a estos tres tipos de días. Es decir, si se observa el comportamiento de la demanda en una hora específica en un rango de días que cobija tanto laborales como festivos, se observan variaciones abruptas propias de distribuciones no normales, lo cual impediría el uso de promedios con el fin de establecer el consumo en esa hora específica. Esto explica por qué fue necesario agrupar los datos de demanda en las tres categorías mencionadas anteriormente.

Debido a que los transformadores evaluados en este proyecto se tomaron de forma aleatoria de diferentes lugares de la ciudad de Bogotá D.C., se concluye que en general el parque de transformadores del nivel de tensión I en efecto se encuentra sobredimensionado. Así pues, es necesario aplicar estrategias tales como la expuesta en este trabajo, de tal manera que el operador de red CODENSA S.A. ESP incremente los activos reconocidos por la CREG, optimizando de esta manera la operación de la red eléctrica en la capital colombiana.

Al considerar que las curvas de carga representan la vida cotidiana de los usuarios, y que, por lo tanto, dependen de las características socioculturales del lugar donde éstos habitan, en este caso Bogotá D.C., es posible concluir que los resultados obtenidos al aplicar la metodología descrita en el presente documento en diferentes ciudades del país, o más aun, entre diferentes países, pueden variar significativamente. 
Aunque es posible determinar parámetros (tasa de mutación, tasa de recombinación, iteraciones y población) para que el algoritmo genético solucione el reordenamiento de conjuntos de transformadores diferentes, el mejor desempeño se obtiene cuando el algoritmo es parametrizado para cada caso. No obstante, el comportamiento de la demanda es una variable incierta que varía en cada sistema de distribución. Sin embargo, a partir del cambio de regulación descrito en la introducción de este documento, se presume que a nivel de Colombia los sistemas de distribución del nivel de tensión I en un futuro cercano se encontrarán sobredimensionados, lo cual permite concluir que bajo esas circunstancias la aplicación de la presente metodología en diferentes regiones del país arrojaría resultados similares a los expuestos.

A observar el modelo implementado y las etapas que lo componen, es posible concluir que independientemente del sistema de distribución en donde se aplique, la metodología descrita en este documento siempre ofrecerá un ordenamiento que produzca los mismos o mejores beneficios económicos a los iniciales. Esto abre el camino para su aplicación en otros países en los cuales la regulación tarifaría vigente castigue a los operadores de red por la ineficiencia de su parque de transformadores, es decir, donde se establezca un límite porcentual de carga, por debajo del cual se ponga algún decremento en el valor final de la energía eléctrica.

Las ganancias asociadas a la ejecución de este proyecto podrían verse incrementadas considerablemente al ser ejecutado en parques de transformación más grandes. Lo anterior teniendo en cuenta el número total de transformadores pertenecientes al nivel de tensión I en los distintos sistemas de distribución del país, y el número de transformadores evaluados en este estudio.

\section{AGRADECIMIENTOS}

Los autores expresan su agradecimiento a la Gerencia de Distribución de la empresa de energía de Bogotá, D.C. CODENSA S.A. ESP por los datos suministrados, y a la Universidad Tecnológica de Pereira por el soporte brindado para el desarrollo de la presente investigación.

\section{REFERENCIAS}

[1] Gerencia de Distribución CODENSA S.A. ESP. "Caracterización de la Demanda en los Diferentes Sectores de la Ciudad de Bogotá”. División Planificación de la Red CODENSA S.A. ESP. 2006.

[2] CREG, Comisión de Regulación de Energía y Gas. Resolución 097 de 2008.

[3] XM Compañía de Expertos de Mercados S.A E.S.P.

[4] IEB Ingeniería Especializada S.A. Consultoría para la Determinación de las Pérdidas de Energía en los Mercados de Comercialización Presentes en el SIN y Definición de Criterios para la Evaluación de Planes de Reducción y/o Mantenimiento de Pérdidas CDP-15207. Septiembre de 2009.

[5] CREG, Comisión de Regulación de Energía y Gas. "Metodología para Definir el Índice de Pérdidas Reconocidas en la Actividad de Distribución". Octubre de 2002.

[6] CREG, Comisión de Regulación de Energía y Gas. "Resolución 082 de 2002". 17 de diciembre de 2002.

[7] Convenio Interadministrativo UTP-CREG. Grupo de Investigación en Planeamiento de Sistemas Eléctricos, Universidad Tecnológica de Pereira. "Apoyo Académico y Soporte Técnico en el Proceso de Implementación de Planes de Reducción de Pérdidas de Energía Eléctrica". 2010.

[8] CODENSA, S.A. ESP. "Estudio de Cargabilidad del Parque de Transformación M.T./B.T". 2002.

[9] ICONTEC. "Norma técnica Colombiana NTC2050". 2001

[10] K. Galindo, P. Cruz and A. Anaya. "Dimensionamiento de Transformadores de Distribución para Sectores Residencial y Comercial Utilizando una Metodología Propuesta, Cumpliendo con el Reglamento Técnico de Instalaciones Eléctricas-RETIE". XII Encuentro Regional Iberoamericano del CIGRÉ. Brasil. 2007.

[11] G. Canavos. "Probabilidad y Estadística". Editorial McGraw-Hill. 1992.

[12] R. Gallego, A. Escobar and E. Toro. "Técnicas Metaheurísticas de Optimización”. Editorial Universidad Tecnológica de Pereira. Pereira, Colombia. 2008. 
[13] F. Glover. "Future Paths for Integer Programming and Links to Artificial Intelligence". Computers \& Operations Research. 13, pp. 533-549. 1986.

[14] T. Crainicand and M. Toulouse. "Handbook of Metaheuristics (Chapter Parallel Strategies for Metaheuristics)". Kluwer Academic Publishers, pp. 475-513. 2003.

[15] S. Kirkpatrick, C. Gelatt and M. Vecchi. "Optimization by Simulated Annealing". Science. Vol. 220, Issue 4598, pp. 671-680. 1983.

[16] T. Feo and M. Resende. "Greedy Randomized Adaptive Search Procedures". Journal of Global Optimization. Vol. 6, pp. 109-133. 1999.

[17] N. Mladenovic and P. Hansen. "Variable Neighborhood Search". Computers Oper. Res. Vol. 24, pp. 1097-1100. 1997.

[18] T. Stützle. "Local Search Algorithms for Combinatorial Problems Analysis, Algorithms and New Applications". Technical
Report, DISKI Dissertationenzur Künstliken Intelligenz. Sankt Augustin, Germany. 1999.

[19] M. Dorigo. "Optimization, Learning and Natural Algorithms". Tesis para optar al Grado de Doctor. Dipartamento di Elettronica. Politecnico di Milano. 1992.

[20] J. Kennedy, R. Eberhart and Y. Shi. "Swarm Intelligence". Morgan Kaufmann Publishers. San Francisco, Estados Unidos. 2001.

[21] L. Fogel, J. Owens and M. Walsh. "Artificial Intelligence Through Simulated Evolution”. John Wiley \& Sons. 1966.

[22] I. Rechenberg. "Evolutions Strategie: Optimierung Technischer System e Nach Prinzipien der Biologischen Evolution". Fromman-Holzboog Verlag. Stuttgart, Alemania. 1973.

[23] J. Holland. "Adaptation in Natural and Artificial Systems". Primera Edición. The MIT Press. Cambridge, Massachusetts. 1975. 